\title{
BIBLIOGRAPHY
}

1. G. Birkhoff and S. MacLane, A survey of modern algebra, rev. ed., Macmillan, New York, 1953.

2. H. Cartan and S. Eilenberg, Homological algebra, Princeton Univ. Press, Princeton, N. J., 1956.

3. R. Crowell and R. Fox, Introduction to knot theory, Ginn, Boston, 1963.

4. R. Crowell, Corresponding group and module sequences, Nagoya Math. J. 19 (1961), 27-40.

5. - The group $G^{\prime} / G^{\prime \prime}$ of a knot group $G$, Duke Math. J. 30 (1963), 349-354.

6. K. Reidemeister, Knotentheorie, Chelsea, New York, 1948.

7. H. Zassenhaus, The theory of groups, Chelsea, New York, 1949.

Dartmouth College

\section{NOTE ON POINTWISE PERIODIC SEMIGROUPS}

\section{ANNE L. HUDSON ${ }^{1}$}

An element $x$ in a semigroup $S$ is said to be periodic if there exists a positive integer $n$ such that $x^{n+1}=x$, and the least such $n, p(x)$, is the period of $x . S$ is pointwise periodic if each $x$ in $S$ is periodic. In [4], A. D. Wallace asks the following question concerning pointwise periodic topological semigroups.

Problem 3: If $S$ is a pointwise periodic semigroup and is topologically an $n$-cell, is it possible that $S \backslash E$ is nonempty and $p(x)>2$ and constant on $S \backslash E$ ?

It will be shown that in a slightly more general situation than that of the above problem, it necessarily follows that $p(x)=2$ on $S \backslash E$.

The following notation will be used throughout this paper. For a semigroup $S, E=\left\{x: x \in S, x^{2}=x\right\}$ and for $e \in E, H(e)$ is the maximal subgroup of $S$ containing the idempotent e. $H=\bigcup\{H(e): e \in E\}$ and functions $y$ and $\theta$ are defined as in [5], that is, for $x \in H, y(x)$ is the idempotent of the unique maximal subgroup to which $x$ belongs and $\theta(x)$ is the inverse of $x$ in this group.

The following theorem will be proved:

THEOREM. Let $S$ be a compact semigroup with the properties:

(1) $S=H$,

(2) for $e \in E, H(e)$ is totally disconnected,

Received by the editors June 7, 1963.

1 The author is the holder of a NATO Postdoctoral fellowship at the University of Tübingen. 
(3) there exists a closed subset $B$ of $S$ such that:

(a) $S \backslash B$ is a connected manifold dense in $S$,

(b) $\operatorname{dim} B<\operatorname{dim} S$.

Then $S$ is a pointwise periodic semigroup and $p(x) \leqq 2$ for each $x$ in $S$.

Before proving this theorem the following lemma will be proved.

LEMмA. Let $S$ be a compact semigroup such that $S=H$ and for each $e$ in $E, H(e)$ is totally disconnected. Then $\operatorname{dim} S=\operatorname{dim} E$.

Proof. Let the equivalence relation $\mathfrak{S}$ be defined as in [5]. Since $S=H$, each $\mathfrak{S}$-class is a group and $S / \mathfrak{S}$ is homeomorphic to $E$ by the restriction to $E$ of the canonical map of $S$ onto $S / \mathfrak{S}$. By Anderson and Hunter [1, Lemma 5, p. 254], it follows that

$$
\operatorname{dim} S \leqq \operatorname{dim} S / \mathfrak{S}+\max \{\operatorname{dim} H(e): e \in E\} .
$$

By assumption each $H(e)$ is totally disconnected, hence zero dimensional. Thus $\operatorname{dim} S \leqq \operatorname{dim} S / \mathfrak{S}=\operatorname{dim} E$ and since $E \subset S$, it follows that $\operatorname{dim} S=\operatorname{dim} E$.

Proof of THEOREM. If it can be shown that $\theta(x)=x$ for each $x$ in $S \backslash B$, then it would follow that $x^{3}=x(\theta(x) x)=x y(x)=x$ for $x \in S \backslash B$. But $T=\left\{x: x^{3}=x\right\}$ is a closed subset of $S$ and if $S \backslash B \subset T$, then $S=T$ since $S \backslash B$ is assumed to be dense in $S$. Thus it suffices to show that $\theta(x)=x$ for $x$ in $S \backslash B$.

To prove $\theta \mid S \backslash B$ is the identity, let $A=\theta(S \backslash B) \cup S \backslash B$ and let $\theta_{0}=\theta \mid A$. Since $\theta^{2}=\theta$ and $\theta$ is a homeomorphism of $S$ onto $S$ [5], $\theta_{0}(A)=A$ is an open subset of $S$. Now let $E_{0}=E \cap S \backslash B$. By the above lemma, $\operatorname{dim} S=\operatorname{dim} E$ which implies that $\operatorname{dim} E_{0}=\operatorname{dim} S=\operatorname{dim} A$, since $\operatorname{dim} B<\operatorname{dim} S$. Hence $E_{0}$ is a nonempty subset of $S \backslash B, \theta_{0}\left(E_{0}\right)$ $=E_{0}$ so that $E_{0} \subset S \backslash B \cap \theta(S \backslash B)$ and $A$ is connected. It will now be shown that $F$, the fixed point set of $\theta_{0}$, has an interior point. From above, $E_{0}$ is a subset of the manifold $A$ and $\operatorname{dim} E_{0}=\operatorname{dim} A$ so that $E_{0}$ contains an interior point. Since $E_{0} \subset F, F$ also has an interior point. Because $A$ is a connected manifold, $\theta_{0}$ is a periodic homeomorphism and $F$ has an interior point, it follows from a theorem of Montgomery and Zippin [3, Theorem 1, p. 223] that $\theta_{0}$ is the identity map. This completes the proof of the theorem.

CoROllary. Let $S$ be a pointwise periodic semigroup on an n-cell. Then $p(x) \leqq 2$ for all $x$ in $S$.

Proof. A pointwise periodic semigroup is the union of groups [2, Theorem 1.9, p. 20], hence $S=H$. Also $H(e)$ is totally disconnected for each $e$ in $E$ since $H(e)$ is a compact periodic group. Letting $B$, in 
the theorem, be the bounding $(n-1)$-sphere of $S$, the corollary follows immediately.

Example (Wallace [6]). Let $I=[0,1]$ be the unit interval with multiplication $x y=\min \{x, y\}$. Let $G=\{e, a\}$ be the two element group with identity $e$ and let $T_{0}=G \times I$ with coordinatewise multiplication. Then $K_{0}=G \times\{0\}$ is an ideal of $T_{0}$ and $T=T_{0} / K_{0}$, the Rees quotient of $T_{0}$ by the ideal $K_{0}$ is a pointwise periodic semigroup whose topological space is a 1-cell.

$T^{n}$, the $n$-fold cartesian product of $T$, with coordinatewise multiplication is a pointwise periodic semigroup on an $n$-cell.

The above example may be varied by letting one of the intervals in the cartesian product be a semigroup with multiplication $x y=x$. In this manner one obtains a pointwise periodic semigroup without a two-sided identity.

\section{REFERENCES}

1. L. W. Anderson and R. P. Hunter, Homomorphisms and dimension, Math. Ann. 147 (1962), 248-268.

2. A. H. Clifford and G. B. Preston, The algebraic theory of semigroups, Math. Surveys No. 7, Amer. Math. Soc., Providence, R. I., 1961.

3. D. Montgomery and L. Zippin, Topological transformation groups, Interscience, New York, 1955.

4. A. D. Wallace, Problems concerning semigroups, Bull. Amer. Math. Soc. 68 (1962), 447-448.

5. - The structure of topological semigroups, Bull. Amer. Math. Soc. 61 (1955), 95-112.

6. - Topological semigroups, Lecture notes, Tulane University, 1961-1962.

UNIVERSITY OF TÜBINGEN 